\title{
Asymptotic Behavior of Solutions of Nonlinear Neutral Delay Forced Impulsive Differential Equations with Positive and Negative Coefficients
}

\author{
Y.Balachandran, S.Pandian, G.Purushothaman, \\ ${ }^{1}$ Department of Mathematics, Presidency College, Chennai-5, Tamilnadu, India \\ ${ }^{2}$ College of arts and science, Thiruvalluvar University, Vandavasi, Tamilnadu, India \\ ${ }^{3}$ Department of Mathematics, St.Joseph's College of Engineering, Chennai-119, Tamilnadu, India
}

\begin{abstract}
Sufficient conditions are obtained for every solution of first order nonlinear neutral delay forced impulsive differential equations with positive and negative coefficients tends to a constant as $t \rightarrow \infty$.

Mathematics Subject Classification [MSC 2010]:34A37
\end{abstract}

Keywords: Asymptotic behavior, neutral, non linear, forced, differential equation, impulse.

\section{Introduction}

In recent years, the theory of impulsive differential equations had obtained much attention and a number of papers have been published in this field. This is due to wide possibilities for their applications in Physics, Chemical technology, Biology and Engineering [1,2].The asymptotic behavior of solutions of various impulsive differential equations are systematically studied (see [4,7-10]). In [10] the authors investigated the asymptotic behavior of solutions of following nonlinear impulsive delay differential equations with positive and negative coefficients

$$
\begin{aligned}
& {[\mathrm{x}(\mathrm{t})+\mathrm{R}(\mathrm{t}) \mathrm{x}(\mathrm{t}-\tau)]^{\prime}+\mathrm{P}(\mathrm{t}) f(\mathrm{x}(\mathrm{t}-\rho))-\mathrm{Q}(\mathrm{t}) f(\mathrm{x}(\mathrm{t}-\sigma))=0, \quad \mathrm{t} \geq \mathrm{t}_{0}, \mathrm{t} \neq \mathrm{t}_{\mathrm{k}}} \\
& \mathrm{x}\left(\mathrm{t}_{\mathrm{k}}\right)=\mathrm{b}_{\mathrm{k}} \mathrm{x}\left(\mathrm{t}_{\mathrm{k}}^{-}\right)+\left(1-\mathrm{b}_{\mathrm{k}}\right)\left(\int_{\mathrm{t}_{\mathrm{k}}-\rho}^{\mathrm{t}_{\mathrm{k}}} \mathrm{P}(\mathrm{s}+\rho) f(\mathrm{x}(\mathrm{s})) \mathrm{ds}-\int_{\mathrm{t}_{\mathrm{k}}-\sigma}^{\mathrm{t}_{\mathrm{k}}} \mathrm{Q}(\mathrm{s}+\sigma) f(\mathrm{x}(\mathrm{s})) \mathrm{ds}\right) \text { for } \mathrm{k}=1,2, \ldots
\end{aligned}
$$

In this paper, we adapt the same technique applied in [10] and obtain the asymptotic behavior of solutions of nonlinear neutral delay forced impulsive differential equations with positive and negative coefficients

\section{Preliminaries} coefficients

Consider the nonlinear neutral delay forced impulsive differential equations with positive and negative

$$
\begin{aligned}
& {[\mathrm{x}(\mathrm{t})+\mathrm{R}(\mathrm{t}) \mathrm{x}(\mathrm{t}-\tau)]^{\prime}+\mathrm{P}(\mathrm{t}) f(\mathrm{x}(\mathrm{t}-\rho))-\mathrm{Q}(\mathrm{t}) f(\mathrm{x}(\mathrm{t}-\sigma))=e(t), \quad \mathrm{t} \geq \mathrm{t}_{0}, \mathrm{t} \neq \mathrm{t}_{\mathrm{k}}} \\
& \mathrm{x}\left(\mathrm{t}_{\mathrm{k}}\right)=\mathrm{b}_{\mathrm{k}} \mathrm{x}\left(\mathrm{t}_{\mathrm{k}}^{-}\right)+\left(1-\mathrm{b}_{\mathrm{k}}\right) \int_{\mathrm{t}_{\mathrm{k}}-\rho}^{\mathrm{t}_{\mathrm{k}}} \mathrm{P}(\mathrm{s}+\rho) f(\mathrm{x}(\mathrm{s})) \mathrm{ds} \\
& \quad-\int_{\mathrm{t}_{\mathrm{k}}-\sigma}^{\mathrm{t}_{\mathrm{k}}} \mathrm{Q}(\mathrm{s}+\sigma) f(\mathrm{x}(\mathrm{s})) \mathrm{ds}+\left(b_{k}-1\right) \int_{\mathrm{t}_{\mathrm{k}}}^{\infty} \mathrm{e}(\mathrm{s}) \mathrm{ds}, \quad k=1,2, \ldots
\end{aligned}
$$

where $\tau>0, \rho \geq \sigma>0,0 \leq \mathrm{t}_{0}<\mathrm{t}_{1}<\cdots<\mathrm{t}_{\mathrm{k}} \cdots$ with $\lim _{\mathrm{k} \rightarrow \infty} \mathrm{t}_{\mathrm{k}}=+\infty, f \in \mathrm{C}(\mathrm{R}, \mathrm{R}), \mathrm{R}(\mathrm{t}) \in \mathrm{PC}\left(\left[\mathrm{t}_{0}, \infty\right), \mathrm{R}\right), \mathrm{P}(\mathrm{t})$, $\mathrm{Q}(\mathrm{t}), \mathrm{e}(\mathrm{t}) \in \mathrm{C}\left(\left[\mathrm{t}_{0}, \infty\right),[0, \infty)\right)$ and $\mathrm{b}_{\mathrm{k}}$ are constants $\mathrm{k}=1,2,3 \ldots, \quad x\left(t_{k}^{-}\right)$denotes the left limit of $\mathrm{x}(\mathrm{t})$ at $\mathrm{t}=\mathrm{t}_{\mathrm{k}}$. With equations (1) and (2), one associates an initial condition of the form

$$
\mathrm{x}\left(\mathrm{t}_{0}+\mathrm{s}\right)=\varphi(\mathrm{s}), \mathrm{s} \in[-\delta, 0], \delta=\max \{\tau, \rho\}
$$

where $\varphi \in \operatorname{PC}([-\delta, 0], \mathrm{R})=\{\varphi:[-\delta, 0] \rightarrow \mathrm{R}$ such that $\varphi$ is continuous everywhere except at the finite number of points $\gamma$ and $\varphi\left(\gamma^{+}\right)$and $\varphi\left(\gamma^{-}\right)$exist with $\left.\varphi\left(\gamma^{+}\right)=\varphi(\gamma)\right\}$.

A real valued function $x(t)$ is said to be a solution of the initial value problem (1)-(3) if

(i) $\mathrm{x}(\mathrm{t})=\varphi\left(\mathrm{t}-\mathrm{t}_{0}\right)$ for $\mathrm{t}_{0}-\delta \leq \mathrm{t} \leq \mathrm{t}_{0}, \mathrm{x}(\mathrm{t})$ is continuous for $\mathrm{t} \geq \mathrm{t}_{0}$ and $\mathrm{t} \neq \mathrm{t}_{\mathrm{k}}, \mathrm{k}=1,2,3, \ldots$

(ii) $[x(t)+R(t) x(t-\tau)]$ is continuously differentiable for $t>t_{0}, t \neq t_{k} t \neq t_{k}+\tau, t \neq t_{k}+\rho, t \neq t_{k}+\sigma$, $\mathrm{k}=1,2,3, \ldots$ and satisfies $(1)$. 
(iii) for $\mathrm{t}=\mathrm{t}_{\mathrm{k}}, \mathrm{x}\left(\mathrm{t}_{\mathrm{k}}^{+}\right)$and $\mathrm{x}\left(\mathrm{t}_{\mathrm{k}}^{-}\right)$exist with $\mathrm{x}\left(\mathrm{t}_{\mathrm{k}}^{+}\right)=\mathrm{x}\left(\mathrm{t}_{\mathrm{k}}\right)$ and satisfies (2).

A solution of (1)-(2) is said to be nonoscillatory if this solution is eventually positive or eventually negative. Otherwise this solution is said to be oscillatory. A more general form of (1)-(2) was considered in [56 ] in which the existence and uniqueness of solutions and stability was studied. The main purpose of this paper is to obtain the sufficient conditions for every solution of (1)-(2) tends to constant as $t \rightarrow \infty$. Our results generalize the results of [10].

\section{Main results}

Theorem 1. Assume that the following conditions hold

(A1) there is a constant $\mathrm{C}>0$ such that $|x| \leq|f(x)| \leq \mathrm{C}|x|, \quad \mathrm{x} \in \mathrm{R}, \mathrm{x} f(\mathrm{x})>0$ for $\mathrm{x} \neq 0$;

(A2) $\mathrm{t}_{\mathrm{k}}-\tau$ is not an impulsive point, $0<\mathrm{b}_{\mathrm{k}}<1$ for $\mathrm{k}=1,2,3, \ldots$ and $\sum_{\mathrm{k}=1}^{\infty}\left(1-\mathrm{b}_{\mathrm{k}}\right)<\infty$;

(A3) $\lim _{\mathrm{t} \rightarrow \infty}|\mathrm{R}(\mathrm{t})|=\mu<1, \mathrm{R}\left(\mathrm{t}_{\mathrm{k}}\right)=\mathrm{b}_{\mathrm{k}} \mathrm{R}\left(\mathrm{t}_{\mathrm{k}}^{-}\right)$and $\int_{t}^{\infty} e(s) d s \rightarrow 0$ as $\mathrm{t} \rightarrow \infty$;

(A4) $\mathrm{H}(\mathrm{t})=\mathrm{P}(\mathrm{t})-\mathrm{Q}(\mathrm{t}+\sigma-\rho)>0$ for $\mathrm{t} \geq \mathrm{t}^{*}=\mathrm{t}_{0}+\rho-\sigma$

(A5) $\lim _{\mathrm{t} \rightarrow \infty} \int_{\mathrm{t}-\rho}^{\mathrm{t}-\sigma} \mathrm{Q}(\mathrm{s}+\sigma) \mathrm{ds}=0$

(A6) $\lim _{\mathrm{t} \rightarrow \infty} \sup \left[\int_{\mathrm{t}-\rho}^{\mathrm{t}+\rho} \mathrm{H}(\mathrm{s}+\rho) \mathrm{d} \mathrm{s}+\frac{\mathrm{Q}(\mathrm{t}+\sigma)}{\mathrm{H}(\mathrm{t}+\rho)} \int_{\mathrm{t}-\rho}^{\mathrm{t}} \mathrm{H}(\mathrm{s}+2 \rho) \mathrm{du}+\mu\left(1+\frac{\mathrm{H}(\mathrm{t}+\tau+\rho)}{\mathrm{H}(\mathrm{t}+\rho)}\right)\right]<\frac{2}{\mathrm{C}}$

then every solution of (1)-(2) tends to a constant as $t \rightarrow \infty$.

Proof. Let $x(t)$ be any solution of (1)-(2) we shall prove that $\lim _{t \rightarrow \infty} x(t)$ exists and is finite. From (1) and (6)

$\left[x(t)+R(t) x(t-\tau)-\int_{t-\rho}^{t} H(s+\rho) f(x(s)) d s-\int_{t-\rho}^{t-\sigma} Q(s+\sigma) f(x(s)) d s+\int_{t}^{\infty} e(s) d s\right]^{\prime}+H(t+\rho) f(x(t))=0$

From (2)

$$
\begin{array}{r}
x\left(t_{k}\right)=b_{k} x\left(t_{k}^{-}\right)+\left(1-b_{k}\right)\left[\int_{t_{k}-\rho}^{t_{k}} P(s+\rho) f(x(s)) d s-\left(\int_{t_{k}-\sigma}^{t_{k}-\rho} Q(s+\sigma) f(x(s)) d s+\int_{t_{k}-\rho}^{t_{k}} Q(s+\sigma) f(x(s)) d s\right)\right] \\
+\left(b_{k}-1\right) \int^{t_{t_{k}}} e(s) d s, \\
=b_{k} x\left(t_{k}^{-}\right)+\left(1-b_{k}\right)\left[\int_{t_{k}-\rho}^{t_{k}-\sigma} Q(s+\sigma) f(x(s)) d s+\int_{t_{k}-\rho}^{t_{k}} H(s+\rho) f(x(s)) d s\right] \\
+\left(b_{k}-1\right) \int_{t_{k}}^{\infty} e(s) d s, \quad k=1,2,3, \ldots,
\end{array}
$$

From (5) and (8) we can choose an $\varepsilon>0$ sufficiently small that $\mu+\varepsilon<1$ and

$\limsup _{t \rightarrow \infty}\left[\int_{t-\rho}^{t+\rho} H(s+\rho) d s+\frac{Q(t+\sigma)}{H(t+\rho)} \int_{t-\rho}^{t} H(s+2 \rho) d s+(\mu+\varepsilon)\left(1+\frac{H(t+\tau+\rho)}{H(t+\rho)}\right)\right]<\frac{2}{C}$

also we select $\mathrm{T}>\mathrm{t}_{0}$ sufficiently large such that $|\mathrm{R}(\mathrm{t})| \leq \mu+\varepsilon$ for $\mathrm{t} \geq \mathrm{T}$

From (4) and (12) we have

$$
R(t) \mid x^{2}(t-\tau) \leq(\mu+\varepsilon) f^{2}(x(t-\tau)), \mathrm{t} \geq \mathrm{T}
$$

Let we introduce three functional

$$
W_{1}(t)=\left[x(t)+R(t) x(t-\tau)-\int_{t-\rho}^{t} H(s+\rho) f(x(s)) d s-\int_{t-\rho}^{t-\sigma} Q(s+\sigma) f(x(s)) d s+\int_{t}^{\infty} e(s) d s\right]^{2}
$$




$$
\begin{aligned}
W_{2}(t) & =\int_{t-\rho}^{t} H(s+2 \rho) \int_{s}^{t} Q(u+\sigma) f^{2}(x(u)) d u d s \\
W_{3}(t) & =\int_{t-\rho}^{t} H(s+2 \rho) \int_{s}^{t} H(u+\rho) f^{2}(x(s)) d u d s+(\mu+\varepsilon) \int_{t-\tau}^{t} H(s+\tau+\rho) f^{2}(x(s)) d s \\
& -2 \int_{t}^{\infty} e(s) \int_{t}^{\infty} H(u+\rho) f(x(u)) d u d s,
\end{aligned}
$$

Using (9) and the inequality $2 a b \leq \mathrm{a}^{2}+\mathrm{b}^{2}$

$$
\begin{aligned}
\frac{d W_{1}}{d t} \leq-H(t+\rho) & {\left[2 x(t) f(x(t))-|R(t)| x^{2}(t-\tau)-|R(t)| f^{2}(x(t))-\int_{t-\rho}^{t} H(s+\rho) f^{2}(x(t)) d s\right.} \\
& \left.-\int_{t-\rho}^{t} H(s+\rho) f^{2}(x(s)) d s-\int_{t-\rho}^{t-\sigma} Q(s+\sigma) f^{2}(x(t)) d s-\int_{t-\rho}^{t-\sigma} Q(s+\sigma) f^{2}(x(s)) d s\right] \\
& -2 H(t+\rho) f(x(t)) \int_{t}^{\infty} e(s) d s \\
\frac{d W_{2}}{d t}= & Q(t+\sigma) f^{2}(x(t)) \int_{t-\rho}^{t} H(s+2 \rho) d s-H(t+\rho) \int_{t-\rho}^{t} Q(s+\sigma) f^{2}(x(s)) d s \\
\leq & Q(t+\sigma) f^{2}(x(t)) \int_{t-\rho}^{t} H(s+2 \rho) d s-H(t+\rho) \int_{t-\rho}^{t-\sigma} Q(s+\sigma) f^{2}(x(s)) d s \\
\frac{d W_{3}}{d t}= & H(t+\rho) f^{2}(x(t)) \int_{t-\rho}^{t} H(s+2 \rho) d s-H(t+\rho) \int_{t-\rho}^{t} H(s+\rho) f^{2}(x(s)) d s \\
& +(\mu+\varepsilon) H(t+\tau+\rho) f^{2}(x(t))-(\mu+\varepsilon) H(t+\rho) f^{2}(x(t-\tau))+2 \int_{t}^{\infty} e(s) H(t+\rho) f(x(t)) d s .
\end{aligned}
$$

Set $\mathrm{W}(\mathrm{t})=\mathrm{W}_{1}(\mathrm{t})+\mathrm{W}_{2}(\mathrm{t})+\mathrm{W}_{3}(\mathrm{t})$; and then using (12) and (13), we obtain

$$
\begin{gathered}
\frac{d W}{d t} \leq-H(t+\rho)\left[2 x(t) f(x(t))-(\mu+\varepsilon) f^{2}(x(t))-f^{2}(x(t)) \int_{t-\rho}^{t} H(s+\rho) d s\right. \\
-f^{2}(x(t)) \int_{t-\rho}^{t} H(s+2 \rho) d s-f^{2}(x(t)) \int_{t-\rho}^{t-\sigma} Q(s+\sigma) d s \\
\left.-\frac{Q(t+\sigma)}{H(t+\rho)} f^{2}(x(t)) \int_{t-\rho}^{t} H(s+2 \rho) d s-(\mu+\varepsilon) f^{2}(x(t)) \frac{H(t+\tau+\rho)}{H(t+\rho)}\right] \\
=-H(t+\rho) f^{2}(x(t))\left[\frac{2 x(t)}{f(x(t))}-\int_{t-\rho}^{t+\rho} H(s+\rho) d s-\frac{Q(t+\sigma)}{H(t+\rho)} \int_{t-\rho}^{t} H(s+2 \rho) d s\right. \\
\left.-\int_{t-\rho}^{t-\sigma} Q(s+\sigma) d s-(\mu+\varepsilon)\left(1+\frac{H(t+\tau+\rho)}{H(t+\rho)}\right)\right]
\end{gathered}
$$

From (4) and (7), we have

$$
\begin{aligned}
\frac{d W}{d t} \leq-H(t+\rho) f^{2}(x(t))\left[\frac{2}{\mathrm{C}}-\left(\int_{t-\rho}^{t+\rho} \mathrm{H}(\mathrm{s}\right.\right. & +\rho) \mathrm{ds}-\frac{\mathrm{Q}(\mathrm{t}+\sigma)}{\mathbf{H}(\mathrm{t}+\rho)} \int_{t-\rho}^{t} \mathrm{H}(\mathrm{s}+2 \rho) \mathrm{ds} \\
& \left.\left.+(\mu+\varepsilon)\left(1+\frac{\mathrm{H}(\mathrm{t}+\tau+\rho)}{\mathrm{H}(\mathrm{t}+\rho)}\right)\right)\right] \leq 0 \quad \mathrm{t} \neq \mathrm{t}_{\mathrm{k}}
\end{aligned}
$$

As $\mathrm{t}=\mathrm{t}_{\mathrm{k}}$ we have

$$
W\left(t_{k}\right)=\left[x\left(t_{k}\right)+R\left(t_{k}\right) x\left(t_{k}-\tau\right)-\int_{t_{k}-\rho}^{t_{k}} H(s+\rho) f(x(s)) d s-\int_{t_{k}-\rho}^{t_{k}-\sigma} Q(s+\sigma) f(x(s)) d s+\int_{t_{k}}^{\infty} e(s) d s\right]^{2}
$$




$$
\begin{aligned}
& \quad+\int_{t_{k}-\rho}^{t_{k}} H(s+2 \rho) \int_{s}^{t_{k}} Q(u+\sigma) f^{2}(x(u)) d u d s+\int_{t_{k}-\rho}^{t_{k}} H(s+2 \rho) \int_{s}^{t_{k}} H(u+\rho) f^{2}(x(u)) d u d s \\
& +(\mu+\varepsilon) \int_{t_{k}-\tau}^{t_{k}} H(s+\tau+\rho) f^{2}(x(s)) d s-2 \int_{t_{k}}^{\infty} e(s) \int_{t_{k}}^{s} H(u+\rho) f(x(u)) d u d s \\
& =b_{k}{ }^{2}\left[x\left(t_{k}^{-}\right)+R\left(t_{k}^{-}\right) x\left(t_{k}^{-}-\tau\right)-\int_{t_{k}-\rho}^{t_{k}} H(s+\rho) f(x(s)) d s-\int_{t_{k}-\rho}^{t_{k}} Q(s+\sigma) f(x(s)) d s+\int_{t_{k}}^{\infty} e(s) d s\right]^{2} \\
& \quad+\int_{t_{k}-\rho}^{t_{k}} H(s+2 \rho) \int_{s}^{t_{k}} Q(u+\sigma) f^{2}(x(u)) d u d s+\int_{t_{k}-\rho}^{t_{k}} H(s+2 \rho) \int_{s}^{t_{k}} H(u+\rho) f^{2}(x(u)) d u d s \\
& \quad+(\mu+\varepsilon) \int_{t_{k}-\tau}^{t_{k}} H(s+\tau+\rho) f^{2}(x(s)) d s-2 \int_{t_{k}}^{\infty} e(s) \int_{t_{k}}^{s} H(u+\rho) f(x(u)) d u d s
\end{aligned}
$$

$=W\left(t_{k}^{-}\right)$

Which together with (7), (8) and (14) we get $H(t+\rho) f^{2}(x(t)) \in L^{1}\left(t_{0}, \infty\right)$

and hence for any $\mathrm{h} \geq 0$ we have $\lim _{t \rightarrow \infty} \int_{t-h}^{t} H(s+\rho) f^{2}(x(s)) d s=0$

On the other hand by (8), (14), (15) we see that $\mathrm{W}(\mathrm{t})$ is eventually decreasing.

$$
\begin{aligned}
0 \leq W_{2}(t) & =\int_{t-\rho}^{t}\left(H(s+2 \rho) \int_{s}^{t} Q(u+\sigma) f^{2}(x(u)) d u\right) d s \\
& \leq \frac{2}{C} \int_{t-\rho}^{t} H(s+\rho) f^{2}(x(u)) d u \rightarrow 0 \text { as } \mathrm{t} \rightarrow \infty
\end{aligned}
$$

Also, $\int_{t-\rho}^{t} H(s+2 \rho) \int_{s}^{t} H(u+\rho) f^{2}(x(u)) d u d s+(\mu+\varepsilon) \int_{t-\tau}^{t} H(s+\tau+\rho) f^{2}(x(s)) d s$

$$
\leq \frac{2}{C} \int_{t-\rho}^{t} H(u+\rho) f^{2}(x(u)) d u+2 \int_{t-\rho}^{t} H(s+\rho) f^{2}(x(s)) d s \rightarrow 0 \text { as } t \rightarrow \infty
$$

and $\int_{t}^{\infty} e(s) \int_{t}^{s} H(u+\rho) f(x(u)) d u d s \leq \int_{t}^{\infty} e(s) \int_{t}^{\infty} H(u+\rho) f(x(u)) d u d s \rightarrow 0$ as $t \rightarrow \infty$

Hence $\lim _{t \rightarrow \infty} W_{1}(t)=\lim _{t \rightarrow \infty} W(t)=\eta$, which is finite. That is

$\lim _{t \rightarrow \infty}\left[x(t)+R(t) x(t-\tau)-\int_{t-\rho}^{t} H(s+\rho) f(x(s)) d s-\int_{t-\rho}^{t-\sigma} Q(s+\sigma) f(x(s)) d s+\int_{t}^{\infty} e(s) d s\right]^{2}=\eta$

Next we shall prove that

$\lim _{t \rightarrow \infty}\left[x(t)+R(t) x(t-\tau)-\int_{t-\rho}^{t} H(s+\rho) f(x(s)) d s-\int_{t-\rho}^{t-\sigma} Q(s+\sigma) f(x(s)) d s+\int_{t}^{\infty} e(s) d s\right]$ exists and is finite.

Let $y(t)=x(t)+R(t) x(t-\tau)-\int_{t-\rho}^{t} H(s+\rho) f(x(s)) d s-\int_{t-\rho}^{t-\sigma} Q(s+\sigma) f(x(s)) d s+\int_{t}^{\infty} e(s) d s$

From (9) we have $y^{\prime}(t)+H(t+\rho) f(x(t))=0$ and from (10) we have

$$
\begin{aligned}
& y\left(t_{k}\right)=b_{k}\left[x\left(t_{k}^{-}\right)+R\left(t_{k}^{-}\right) x\left(t_{k}-\tau\right)-\int_{t_{k}-\rho}^{t_{k}} H(s+\rho) f(x(s)) d s+\int_{t_{k}-\rho}^{t_{k}-\sigma} Q(s+\sigma) f(x(s)) d s+\int_{t_{k}}^{\infty} e(s) d s\right] \\
& =b_{k} y\left(t_{k}^{-}\right)
\end{aligned}
$$


Therefore system (9)-(10) can be rewritten as $y^{\prime}(t)+H(t+\rho) f(x(t))=0, t \geq t_{0}, t \neq t_{k}$

$$
y\left(t_{k}\right)=b_{k} y\left(t_{k}^{-}\right) \quad \mathrm{k}=1,2, \ldots
$$

In view of (18) we have $\lim _{t \rightarrow \infty} y^{2}(t)=\eta$.

If $\eta=0$, then $\lim _{t \rightarrow \infty} y^{2}(t)=0$.

If $\eta>0$, then there exists a sufficiently large $T_{1}$ such that $y(t) \neq 0$ for any $t>T_{1}$. Otherwise there is a sequence $\tau_{1}, \tau_{2}, \tau_{3}, . . \tau_{k}, .$. with $\lim _{k \rightarrow \infty} \tau_{k}=+\infty$ such that $\mathrm{y}\left(\tau_{\mathrm{k}}\right)=0$ so $\mathrm{y}^{2}\left(\tau_{\mathrm{k}}\right)=0$ as $\mathrm{k} \rightarrow \infty$. This is contradiction to $\eta>$ 0 .Therefore for $t_{k}>T_{1}, t \in\left[t_{k}, t_{k+1}\right)$ we have $y(t)>0$ or $y(t)<0$ because $y(t)$ is continuous on $\left[t_{k}, t_{k+1}\right)$. Without loss of generality we assume that $\mathrm{y}(\mathrm{t})>0$ on $\mathrm{t} \in\left[\mathrm{t}_{\mathrm{k}}, \mathrm{t}_{\mathrm{k}+1}\right)$, it follows that $\mathrm{y}\left(\mathrm{t}_{\mathrm{k}+1}\right)=b_{k+1} y\left(t_{k+1}^{-}\right)>0$, thus $\mathrm{y}(\mathrm{t})>0$ on $\left[\mathrm{t}_{\mathrm{k}+1}, \mathrm{t}_{\mathrm{k}+2}\right)$. By induction, we can conclude that $\mathrm{y}(\mathrm{t})>0$ on $\left[\mathrm{t}_{\mathrm{k}}, \infty\right)$.

From (20) we have

$\lim _{t \rightarrow \infty} y(t)=\lim _{t \rightarrow \infty}\left[x(t)+R(t) x(t-\tau)-\int_{t-\rho}^{t} H(s+\rho) f(x(s)) d s-\int_{t-\rho}^{t-\sigma} Q(s+\sigma) f(x(s)) d s+\int_{t}^{\infty} e(s) d s\right]=\lambda$

where $\lambda=\sqrt{\eta}$ and is finite.

In view of (19) we have $\int_{t-\rho}^{t} H(s+\rho) f(x(s)) d s=y(t-\rho)-y(t)+\sum_{t-\rho<t_{k}<t}\left[y\left(t_{k}\right)-y\left(t_{k}^{-}\right)\right]$

$$
\begin{aligned}
& =y(t-\rho)-y(t)+\sum_{t-\rho<t_{k}<t}\left[b_{k} y\left(t_{k}^{-}\right)-y\left(t_{k}^{-}\right)\right] \\
& =y(t-\rho)-y(t)-\sum_{t-\rho<t_{k}<t}\left(1-b_{k}\right) y\left(t_{k}^{-}\right)
\end{aligned}
$$

Let $\mathrm{t} \rightarrow \infty \sum_{k=1}^{\infty}\left(1-b_{k}\right)<\infty$, we have $\lim _{t \rightarrow \infty} \int_{t-\rho}^{t} H(s+\rho) f(x(s)) d s=0$

By condition $\lim _{t \rightarrow \infty} \int_{t}^{\infty} e(s) d s=0$ and from (21), we have

$$
\lim _{t \rightarrow \infty}\left[x(t)+R(t) x(t-\tau)-\int_{t-\rho}^{t-\sigma} Q(s+\sigma) f(x(s)) d s\right]=\lambda
$$

Next we shall prove that $\lim _{t \rightarrow \infty}[x(t)+R(t) x(t-\tau)]$ exists and is finite. To prove this, first prove that $|x(t)|$ is bounded. If $|x(t)|$ is unbounded then there is a sequence $\left\{\mathrm{s}_{\mathrm{n}}\right\}$ such that $\mathrm{s}_{\mathrm{n}} \rightarrow \infty, \quad\left|x\left(s_{n}^{-}\right)\right| \rightarrow \infty$ as $\mathrm{n} \rightarrow \infty$ and $\left|\mathrm{x}\left(\mathrm{s}_{\mathrm{n}}^{-}\right)\right|=\operatorname{Sup}_{\mathrm{t}_{0} \leq t \leq \mathrm{s}_{\mathrm{n}}}|\mathrm{x}(\mathrm{t})|$ where if $\mathrm{s}_{\mathrm{n}}$ is not an impulsive point then $x\left(s_{n}^{-}\right)=x\left(s_{n}\right)$. Thus from (7) we have

$$
\begin{aligned}
\mid x\left(s_{n}^{-}\right)+R\left(s_{n}^{-}\right) x\left(s_{n}-\tau\right)- & \int_{s_{n}-\rho}^{s_{n}-\sigma} Q(s+\sigma) f(x(s)) d s \mid \\
& \geq\left|x\left(s_{n}^{-}\right)\right|\left(1-(\mu+\varepsilon)-M \int_{s_{n}-\rho}^{s_{n}-\sigma} Q(s+\sigma) d s\right) \rightarrow \infty \text { as } n \rightarrow \infty
\end{aligned}
$$

which contradicts (23). So $|x(t)|$ is bounded. Also by (7) we obtain

$0 \leq\left|\int_{t-\rho}^{t-\sigma} Q(s+\sigma) f(x(s)) d s\right| \leq \int_{t-\rho}^{t-\sigma} Q(s+\sigma)|f(x(s))| d s \leq M \int_{t-\rho}^{t-\sigma} Q(s+\sigma)|x(s)| d s \rightarrow 0$ as $t \rightarrow \infty$

which together with (23) gives $\lim _{t \rightarrow \infty}[x(t)+R(t) x(t-\tau)]=\lambda$

Next we prove that $\lim _{t \rightarrow \infty} x(t)$ exists and finite . 
If $\mu=0$ then $\lim _{t \rightarrow \infty} x(t)=\lambda$ which is finite.

If $0<\mu<1$ then $\mathrm{R}(\mathrm{t})$ is eventually positive or negative and also we can find a sufficiently large $\mathrm{T}_{2}$ such that $|R(t)|<1$ for $\mathrm{t}>\mathrm{T}_{2}$. Set $\lim _{t \rightarrow \infty} \sup x(t)=L, \liminf _{t \rightarrow \infty} x(t)=l$, then we can choose two sequences $\left\{\mathrm{a}_{\mathrm{n}}\right\}$ and $\left\{\mathrm{b}_{\mathrm{n}}\right\}$ such that $\mathrm{a}_{\mathrm{n}} \rightarrow \infty, \mathrm{b}_{\mathrm{n}} \rightarrow \infty$ as $\mathrm{n} \rightarrow \infty$ and $\lim _{n \rightarrow \infty} x\left(a_{n}\right)=L, \lim _{n \rightarrow \infty} x\left(b_{n}\right)=l$. Since $|R(t)|<1$ for $\mathrm{t}>\mathrm{T}_{2}$, we have the following two possible cases.

Case 1. If $0<\mathrm{R}(\mathrm{t})<1$ for $\mathrm{t}>\mathrm{T}_{2}$ then $\lim _{\mathrm{n} \rightarrow \infty}\left[x\left(a_{n}\right)+R\left(a_{n}\right) x\left(a_{n}-\tau\right)\right] \geq L+\mu l$ and

$$
\lim _{n \rightarrow \infty}\left[x\left(b_{n}\right)+R\left(b_{n}\right) x\left(b_{n}-\tau\right)\right] \leq l+\mu L
$$

Therefore $\mathrm{L}+\mu l \leq l+\mu \mathrm{L}$. i.e.). $\mathrm{L} \leq l$. But $L \geq l$, it follows that $L=l$. Hence $\mathrm{L}=l=\lambda(1+\mu)$. which shows that $\lim _{\mathrm{t} \rightarrow \infty} \mathrm{x}(\mathrm{t})$ exists and finite.

Case 2. If $-1<\mathrm{R}(\mathrm{t})<0$ for $\mathrm{t}>\mathrm{T}_{2}$ then $\lim _{n \rightarrow \infty} x\left(a_{n}\right)=\lim _{n \rightarrow \infty}\left[x\left(a_{n}\right)+R\left(a_{n}\right) x\left(a_{n}-\tau\right)-R\left(a_{n}\right) x\left(a_{n}-\tau\right)\right]$ therefore $l=\lambda+\mu l$. i.e. $l=\lambda(1-\mu)$.

Similarly $\lim _{n \rightarrow \infty} x\left(b_{n}\right)=\lim _{n \rightarrow \infty}\left[x\left(b_{n}\right)+R\left(b_{n}\right) x\left(b_{n}-\tau\right)-R\left(b_{n}\right) x\left(b_{n}-\tau\right)\right]$

Therefore $\mathrm{L}=\lambda+\mu \mathrm{L}$ i.e. $) \mathrm{L}=\lambda(1-\mu)$.Finally we get, $\mathrm{L}=l=\lambda /(1-\mu)$. This shows that $\lim _{t \rightarrow \infty} x(t)$ exists and finite.

Theorem 2. Assume that the conditions in theorem (1) hold, then every oscillatory solution of (1)-(2) tends to zero as $\mathrm{t} \rightarrow \infty$.

Theorem 3. The conditions in theorem (1) together with

(i) for any $\alpha>0$ there exists $\beta>0$ such that $|f(x)| \geq \beta$ for $|x| \geq \alpha$ and

(ii) $\int_{t_{0}}^{\infty} H(t) d t=\infty$

(26) imply that every solution of (1) -(2) tends to zero as $t \rightarrow \infty$.

Proof. By theorem (2) we only have to prove that every nonoscillatory solution of (1) tends to zero as $t \rightarrow \infty$.Let $\mathrm{x}(\mathrm{t})$ be an eventually positive solution of (1). We shall prove $\lim _{t \rightarrow \infty} x(t)=0$. By theorem (1) we rewrite (1)-(2) in the form $y^{\prime}(t)+H(t+\rho) f(x(t))=0$.

Integrating from $\mathrm{t}_{0}$ to $t$ on both sides we get

$$
\int_{t_{0}}^{t} H(t+\rho) f(x(s)) d s=y\left(t_{0}\right)-y(t)-\sum_{t_{0}<t_{k}<t}\left(1-b_{k}\right) y\left(t_{k}^{-}\right)
$$

Using $\sum_{k=1}^{\infty}\left(1-b_{k}\right)<\infty$ and (21) we get $\int_{t_{0}}^{\infty} H(t+\rho) f(x(s)) d s<\infty$

which together with (26) we get $\lim _{t \rightarrow \infty} \inf f\left(x(t)=0\right.$. We shall prove that $\liminf _{t \rightarrow \infty} x(t)=0$.Let $\left\{s_{m}\right\}$ be such that $\mathrm{s}_{\mathrm{m}} \rightarrow \infty$ as $\mathrm{m} \rightarrow \infty$ and $\lim _{m \rightarrow \infty} \inf f\left(x\left(s_{m}\right)\right)=0$ we must have $\lim _{m \rightarrow \infty} \inf x\left(s_{m}\right)=M=0$. In fact, if $\mathrm{M}>0$, then there is a subsequence $\left\{s_{m_{k}}\right\}$ such that $x\left(s_{m_{k}}\right) \geq M / 2$ for sufficiently large k. By (25) we have $f\left(x\left(s_{m_{k}}\right) \geq \xi\right.$ for some $\xi>0$ and sufficiently large $\mathrm{k}$, which yields a contradiction because $\liminf _{k \rightarrow \infty} f\left(x\left(s_{m_{k}}\right)\right)=0$. Therefore by Theorem 1, $\lim _{t \rightarrow \infty} \inf x(t)=0$ holds and hence $\lim _{t \rightarrow \infty} x(t)=0$.

Remark 1. When $\mathrm{e}(\mathrm{t})=0$ the results of this paper reduce to the results of [10].

Remark 2. When $\mathrm{R}(\mathrm{t})=0, \mathrm{Q}(\mathrm{t})=0$ and $\mathrm{e}(\mathrm{t})=0$ the results of the present paper reduce to the results of [9]. 
Remark 3. When all $b_{k}=1$ and $f(x)=x$, equation (1)-(2) reduced to the differential equation without impulses whose asymptotic behavior of solutions discussed in [3]

Consider the following impulsive differential equation

\section{Example}

$$
\begin{gathered}
{\left[\mathrm{x}(\mathrm{t})+\left(\frac{\mathrm{t}}{8 \mathrm{k}}\right) \mathrm{x}\left(\mathrm{t}-\frac{1}{2}\right)\right]^{\prime}+\left(\frac{2}{(\mathrm{t}-1)^{2}}\right)\left[1+\sin ^{2} \mathrm{x}(\mathrm{t}-2)\right] \mathrm{x}(\mathrm{t}-2)-\frac{1}{\mathrm{t}^{2}}\left[1+\sin ^{2} \mathrm{x}(\mathrm{t}-1)\right] \mathrm{x}(\mathrm{t}-1)=e^{-t}, \mathrm{t} \geq 2, \mathrm{t} \neq \mathrm{k}} \\
\mathrm{x}(\mathrm{k})=\left(\frac{\mathrm{k}^{2}-1}{\mathrm{k}^{2}}\right) \mathrm{x}\left(\mathrm{t}_{\mathrm{k}}^{-}\right)+\left(\frac{1}{\mathrm{k}^{2}}\right)\left(\int_{\mathrm{k}-2}^{\mathrm{k}} \frac{2}{(\mathrm{~s}+1)^{2}}\left[1+\sin ^{2} \mathrm{x}(\mathrm{s})\right] \mathrm{x}(\mathrm{s}) \mathrm{ds}-\int_{\mathrm{k}-1}^{\mathrm{k}} \frac{1}{(\mathrm{~s}+1)^{2}}\left[1+\sin ^{2} \mathrm{x}(\mathrm{s})\right] \mathrm{x}(\mathrm{s}) \mathrm{ds}\right) \\
-\frac{1}{k^{2}} \int_{k}^{\infty} e(s) d s \text { for } \mathrm{k}=1,2,3, \ldots
\end{gathered}
$$

Here $\mathrm{P}(\mathrm{t})=\frac{2}{(\mathrm{t}-1)^{2}}, \mathrm{Q}(\mathrm{t})=\frac{1}{\mathrm{t}^{2}}, \mathrm{R}(\mathrm{t})=\frac{\mathrm{t}}{8 \mathrm{k}}, f(\mathrm{x})=\left[1+\sin ^{2} \mathrm{x}\right] \mathrm{x}, \tau=1 / 2, \rho=2, \sigma=1, \mathrm{~b}_{\mathrm{k}}=\frac{\mathrm{k}^{2}-1}{\mathrm{k}^{2}}$

The above equation satisfies all the conditions of Theorem 1 . Therefore, every solution of this equation tends to constant as $\mathrm{t} \rightarrow \infty$.

\section{References}

[1] V. Lakshmikantham, D.D. Bainov, P.S. Simeonov, Theory of Impulsive Differential Equations, World Scientific, Singapore, 1989

[2] D.D. Bainov and P.S. Simeonov, Impulsive Differential Equations: Periodic Solutions and Applications, Longman, Harlow, 1993.

[3] J.H. Shen and J.S. Yu, Asymptotic behavior of solutions of neutral differential equations with positive and negative coefficients, J. Math. Anal. Appl., 195(1995), 517-526.

[4] Aimin Zhao and Jurang Yan, Asymptotic behavior of solutions of impulsive delay differential equations, J. Math. Anal. Appl., 201(1996), 943-954.

[5] Jianhua Shen, Zhiguo Luo, Xinzhi Liu, Impulsive stabilization for functional differential equations via Liapunov functional, J. Math. Anal. Appl. 240 (1999) 1-15.

[6] Xinzhi Liu, G. Ballinger, Existence and continuability of solutions for differential equations with delays and state dependent impulses, Nonlinear Anal. 51 (2002) 633-647.

[7] Gengping Wei and Jianhua Shen, Asymptotic behavior of solutions of nonlinear impulsive delay differential equations positive and negative coefficients, Math. \& Comp. Modeling, 44(2006), 1089-1096.

[8] Jiowan Luo and Lokenath Debonath, Asymptotic behavior of solutions of forced nonlinear neutral delay differential equations with impulses J. Appl. Math. \& Computing, 12(2003),39-47.

[9] Jianhua Shen and Y.Liu, Asymptotic behavior of solutions of nonlinear delay differential equations with impulses, Appl. Math. and Comput., 213(2009), 449-454.

[10] S. Pandian, G. Purushothaman and Y. Balachandran, Asymptotic behavior of solutions nonlinear neutral delay impulsive differential equations with positive and negative coefficients, Far East Journal of Mathematical Sciences, 51(2) (2011), 165 --178. 